\title{
Erläuterungen zu den neuen Richtlinien zur ärztlichen Untersuchung von Feuerwehrleuten SFV
}

Christian Hug

\footnotetext{
Der SFV nimmt als Zusammenschluss aller Orts-, Berufs- und Betriebsfeuerwehren der Schweiz und des Fürstentums Liechtenstein die Interessen der Feuerwehren wahr und vertritt sie nach aussen. Unter anderem unterhält der SFV eine Hilfskasse, die im Falle von Unfällen oder Krankheiten im Zusammenhang mit dem Feuerwehrdienst bei Lücken im Bereich der obligatorischen Versicherungen subsidiäre Leistungen erbringt.

** Selbstverständlich gelten alle Aussagen auch für Ärztinnen sowie weibliche AdF, die heute Seite an Seite bis auf Kommandantinnenstufe mit ihren männlichen Kollegen Feuerwehrdienst leisten.
}

\section{Korrespondenz:}

Dr. med. Christian Hug

Innere Medizin FMH

Vertrauensarzt SFV

Mayweg 5

CH-3007 Bern

Tel. 0313721010

zaeringer@bluewin.ch

\begin{abstract}
Einleitung
Die Feuerwehr übernimmt neben den Partnerorganisationen Sanität, Polizei, Zivilschutz und Armee wichtige Aufgaben im Bevölkerungsschutz. Diese reichen von Routineeinsätzen bis zur Bewältigung von Grossereignissen. Von den Angehörigen der Feuerwehr (AdF) werden in unserem hochtechnisierten Umfeld zunehmend spezifische Kenntnisse und Fähigkeiten verlangt. Bis auf einige Berufsfeuerwehren sind die Feuerwehren als Milizsystem aufgebaut. Trotzdem wird von allen AdF jederzeit professionelles Handeln erwartet.
\end{abstract}

\section{Risiken für die Angehörigen der Feuerwehr}

Neben diesem permanenten Leistungsdruck sind die AdF oft auch massiven körperlichen und psychischen Belastungen ausgesetzt. Im Einsatz können unerwartet verschiedenste Gefahren auftreten: Unfälle im Strassenverkehr (Alarmfahrten), Verletzung durch Einsturz, Explosion, Fall usw., chemische und radioaktive Produkte, gefährliche Tiere sowie toxischer Rauch und Hitze. Brände machen zwar längstens nicht die Mehrzahl der Einsätze der Feuerwehr aus, sind aber mit spezifischen und den für die AdF wichtigsten Risiken verbunden. Mit dem systematischen Gebrauch der Atemschutzgeräte (ASG) stellen Rauch und toxische Gase heute keine unmittelbare Gesundheitsgefährdung mehr dar. Arbeiten in voller Brandschutzausrüstung (Atemschutzgerät, isolierende Brandschutzbekleidung, Helm, Stiefel, Lösch- und Rettungsgeräte) sind körperlich sehr anstrengend, und die Hitzebelastung kann enorm werden. Die maximale Herzfrequenz wird dabei rasch erreicht, und es besteht die Gefahr eines Schwächezustandes, bei Jungen durch eine bisher unentdeckte Kardiopathie (HOCM), bei Älteren durch eine Arrhythmie bzw. Myokardischämie. Zudem können eine hitzebedingte Vasodilatation zusammen mit einer Dehydratation durch exzessives Schwitzen bzw. eine Hyperthermie zum Kollaps führen. Diese an sich meist beherrschbaren Probleme können, wenn sie mitten in einem Brand auftre- ten, deletär sein und nicht nur den einzelnen, sondern den ganzen Atemschutztrupp gefährden. Einsätze in Atemschutzgeräten sind auch psychisch sehr belastend: Der mit dem Einsatz verbundene Stress (enge Verhältnisse, fehlende Sicht, Lärm, Konfrontation mit Opfern, Erfolgsdruck) kann Atemschutzgeräteträger an die Grenzen ihrer Belastbarkeit bringen. Klaustrophobie oder Panikattacken können in solchen Situationen äusserst gefährlich sein (z. B. Atemschutzmaske vom Gesicht reissen).

Die Überprüfung des Gesundheitszustandes ist zwar nicht gesetzlich vorgeschrieben, wird aber vom Schweizerischen Feuerwehrverband* verlangt (SFV, www.swissfire.ch).

\section{Überlegungen und Gründe zur Revision der Richtlinien}

- Gegenüber den bisherigen «Weisungen für die ärztliche Untersuchung von Feuerwehrleuten $S F V »$ muss aufgrund der neueren Literatur vor allem der Erfassung der körperlichen Leistungsfähigkeit und des kardiovaskulären Risikoprofils mehr Bedeutung beigemessen werden.

- Wie oben erläutert, sind AdF, im besonderen Atemschutzgeräteträger (ASGT), oft physischen und psychischen Maximalbelastungen ausgesetzt.

- Daher soll eine sorgfältige medizinische Untersuchung Probanden** erkennen, die sich, ihre Kameraden oder den Erfolg des Einsatzes aus gesundheitlichen Gründen gefährden.

- Die ärztliche Untersuchung soll der Funktion der AdF bzw. den zu erwartenden Risiken angepasst sein.

- Die Untersuchung soll mit möglichst einfachen Mitteln ein möglichst aussagekräftiges Resultat ergeben, kostengünstig sein und den föderalistischen und dezentralen geographischen Strukturen unseres Landes gerecht werden.

- Bei der Beurteilung darf keine Unterscheidung zwischen Berufsleuten und Milizangehörigen oder zwischen Übungsdienst und Einsatz gemacht werden. 
- Je besser der Arzt die Gefahren des Feuerwehrdienstes und die sich daraus ergebenden Anforderungen kennt, desto kompetenter kann er die AdF bezüglich Tauglichkeit beurteilen. Es gibt keine Spezialisierung bzw. keinen Fähigkeitsausweis «Feuerwehrarzt». Trotzdem ist es sehr wünschenswert, dass Kollegen, die AdF auf ihre Tauglichkeit hin untersuchen, sich aktiv mit dieser Materie befassen bzw. dass die Feuerwehren ihre Ärzte nach diesen Gesichtspunkten auswählen.

- Ein weiteres Ziel der Revision war es, für AdF mit Fahrausweis Kategorie C (Lastwagen) bzw. C1 (schwere Feuerwehrfahrzeuge) die Doppelspurigkeit der Untersuchung beim Feuerwehrarzt und beim Vertrauensarzt des Strassenverkehrsamtes zu vermeiden.

\section{Die neuen Richtlinien}

Unfälle oder Erkrankungen im Feuerwehrdienst sind zum Glück selten. Zudem gibt es in der Schweiz zu diesem Thema keine guten medizinischen Daten. Man muss sich also an möglichen Gefahren, an Richtlinien zu ähnlichen Tätigkeiten, an aktuellen Guidelines und an ausländischen Richtlinien und Statistiken orientieren. Die vorliegenden neuen Richtlinien wurden in diesem Sinne von einer Arbeitsgruppe bestehend aus vier Ärzten ${ }^{\star * *}$ erarbeitet. An ASGT werden besonders hohe physische Anforderungen gestellt, weshalb für sie ein periodischer Leistungstest empfohlen wird. Dem mit zunehmendem Alter steigenden kardiovaskulären Risiko wird speziell Beachtung geschenkt Daraus ergibt sich ein vierstufiger Untersuchungsgang, wobei wie überall in der Medizin den kostengünstigen Instrumenten Anamnese und Status die wichtigste Bedeutung zukommen sollte.

Aufgrund der obgenannten Überlegungen wurde ein modularer Aufbau gewählt (Abb. 1).

- Bei Eintritt in den allgemeinen Feuerwehrdienst wird von den AdF ein detaillierter Gesundheitsfragebogen ausgefüllt und unter Wahrung des Arztgeheimnisses dem Feuerwehrarzt zur Überprüfung der Tauglichkeit zugestellt. Damit muss der Feuerwehrarzt nur noch bei Bedarf ergänzende anamnestische Angaben erheben. Die AdF übernehmen mit ihrer Unterschrift die Verantwortung für die von ihnen gemachten Angaben.

- Eine körperliche Untersuchung erfolgt, wenn aufgrund des Fragebogens bzw. der Anamnese dies zur Beurteilung der Tauglichkeit für den allgemeinen Feuerwehrdienst notwendig erscheint.

- Nebst dem Ausfüllen des Fragebogens ist eine detaillierte körperliche Untersuchung beim
Eintritt in den Atemschutzdienst, bei den periodischen Kontrolluntersuchungen der ASGT sowie bei Untersuchungen von Fahrern von schweren Motorfahrzeugen der Kategorie C bzw. C1, sofern diese vom Feuerwehrarzt durchgeführt werden, nötig.

- Zusätzlich wird bei ASGT über 40 Jahren das kardiovaskuläre Risiko gemäss dem Risikoscore der European Society of Cardiology (ESC) evaluiert. Dazu ist die Bestimmung eines Gelegenheitswertes für Glukose und Gesamtcholesterin notwendig.

- Falls dabei ein erhöhtes Risiko festgestellt wird und die Tauglichkeit des ASGT belegt werden muss, kann dies mittels Ergometrie (Belastungs-EKG oder Laufbandergometrie) erfolgen.

- Zudem kann es nötig sein, bei speziellen Fragestellungen oder grenzwertigen Befunden weiterführende Untersuchungen (Labor, Bildgebung, Funktionstests) oder den Zuzug eines entsprechenden Spezialarztes zu veranlassen.

- Für ASGT ist ausserdem ein jährlicher sportlicher Leistungstest mit klaren Leistungslimiten vorgesehen. Wird im Rahmen der ärztlichen Untersuchung ohnehin ein Belastungs-EKG durchgeführt, kann dieses als Leistungstest gelten.

Details zum Untersuchungsgang, zu Tauglichkeitskriterien und zur Leistungstestung sind auf der Homepage des Schweizerischen Feuerwehrverbandes (SFV) (www.swissfire.ch) unter Hilfskasse zu finden. Dort können der Fragebogen für die/den Angehörige/n der Feuerwehr, das Statusblatt, das Zeugnisformular, die Richtlinien für die ärztliche Untersuchung von Feuerwehrleuten SFV sowie Appendizes und Quellenhinweise heruntergeladen werden. Die genannten Unterlagen können auch beim Schweizerischen Feuerwehrverband bestellt werden (Morgenstrasse 1, Postfach, 3073 Gümligen, Tel. 03195881 18, Fax 03195881 11).

\section{Untersuchung von Fahrern Kat. C und C1}

Bisher bestand eine Doppelspurigkeit bei den ärztlichen Untersuchungen von ASGT, die zugleich Fahrer der Kategorie C bzw. C1 sind. Sofern der Feuerwehrarzt nicht zugleich Vertrauensarzt des entsprechenden kantonalen Strassenverkehrsamtes war, musste sich dieser AdF auch noch bei einem solchen untersuchen lassen, was doppelte Kosten verursachte. Im Bestreben, dies zu vermeiden, wurde die feuerwehrärztliche Untersuchung, die viel weiter geht als die Untersuchung für Fahrer Kategorie C und 


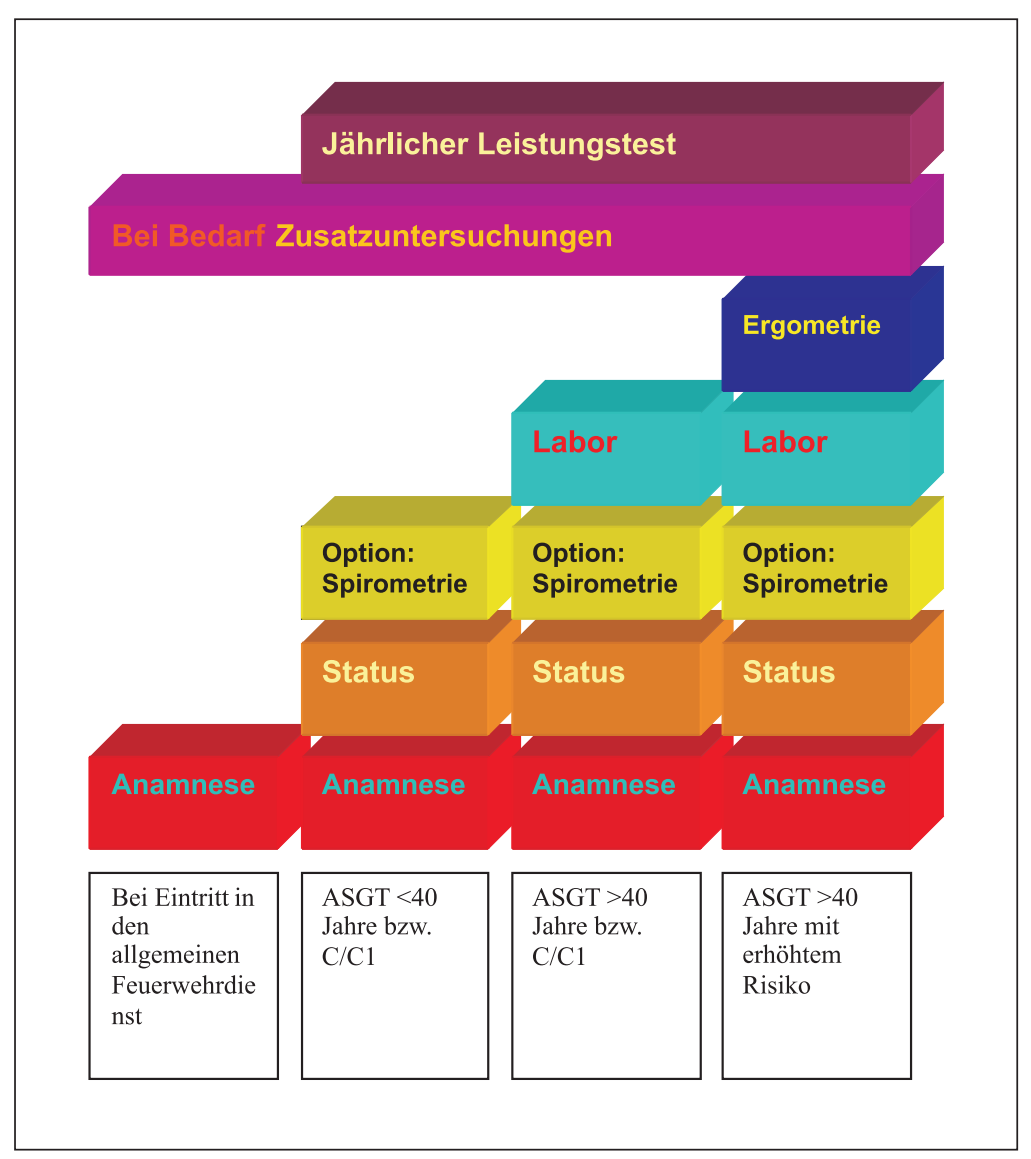

C1, genau auf letztere abgestimmt. Diese Übereinstimmung wurde durch Dr. med. R. Seeger (stellvertretender Leiter der Abteilung Verkehrsmedizin und klinische Forensik des Institutes für Rechtsmedizin der Universität Zürich) sowie durch das Bundesamt für Strassen (ASTRA) mit Brief vom 5. Oktober 2007 folgendermassen bestätigt: «Wir haben Ihre Unterlagen geprüft und können Ihnen mitteilen, dass sie mit dem in der Verkehrszulassungsverordnung vorgeschriebenen Untersuchungsgang vereinbar sind.» Es ist nun an den Feuerwehren bzw. den kantonalen Feuerwehrverbänden, mit den entsprechenden Strassenverkehrsämtern zu klären, ob die Untersuchung durch den Feuerwehrarzt, sofern dieser nicht ohnehin Vertrauensarzt des Strassenverkehrsamtes ist, als Untersuchung für die Kategorie C/C1 anerkannt wird.

\section{Verantwortlichkeiten}

Die Überprüfung der Tauglichkeit für die vielfältigen und belastenden Tätigkeiten der AdF ist einerseits eine ärztliche Aufgabe und das Thema dieses Artikels. Mit dem beschriebenen medizinischen Untersuchungsgang können jedoch nicht sämtliche Aspekte abgedeckt werden. Daher kommt auch Kursleitern, Vorgesetzten und Sachverständigen eine wichtige Funktion zu; nur sie können das Verhalten des AdF im Einsatz und im Umgang mit der konkreten Gefahr beurteilen und sollen bei Hinweisen auf eine medizinisch bedingte Einschränkung der Tauglichkeit eine Untersuchung beim Feuerwehrarzt verlangen.

\section{Zusammenfassung}

Die wichtigsten Neuerungen gegenüber den bisherigen «Weisungen für die ärztliche Untersuchung von Feuerwehrleuten $S F V$ » sind:

- der Gesundheitsfragebogen für alle Anwärter für den Feuerwehrdienst;

- die vertiefte Evaluation des kardiovaskulären Risikoprofils;

- der jährliche Leistungstest für alle ASGT;

- die Empfehlung, dass die Feuerwehren mit Ärzten zusammenarbeiten, die sich vertieft mit dem Thema «medizinische Tauglichkeit der AdF» auseinandersetzen. Diese Ärzte sollen auch für die Sanitätsausbildung und bei Einsätzen als Fachpersonen beigezogen werden können.

\section{Dank}

Nebst den vielen Rückmeldungen von Kolleginnen und Kollegen im Gefolge des zweiten Schweizerischen Feuerwehrkongresses im Jahr 2005 in Aesch BL hat auch die wertvolle Mitarbeit von Dr. med. R. Seeger, Institut für Rechtsmedizin der Universität Zürich-Irchel, Verkehrsmedizin und klinische Forensik, und von Dr. med. Toni Held, Facharzt für Allgemeinmedizin FMH, speziell Sportmedizin, Hirslanden, Sportklinik Birshof, Reinacherstrasse 28, 4142 Münchenstein, ganz massgelblich zum Gelingen dieser Revision beigetragen. Ihnen allen sei ein grosser Dank ausgesprochen.

Am Donnerstag, dem 18. September 2008, wird in Bern ein Fortbildungstag für Feuerwehrärzte mit dem Hauptthema der neuen «Richtlinien für die ärztliche Untersuchung von Feuerwehrleuten SFV» stattfinden. Eine Einladung wird demnächst in der Schweizerischen Ärztezeitung erscheinen. 